\title{
DENSIDADE DE OVIPOSIÇÃO, E QUANTIFICAÇÃO DE LARVAS E PUPAS DE Mansonia BLANCHARD, 1901 (DIPTERA: CULICIDAE), EM Eichhornia crassipes SOLMS. E Pistia stratiotes LINN. NA ILHA DA MARCHANTARIA, AMAZÔNIA CENTRAL. ${ }^{1}$
}

\author{
Ruth Leila Menezes FERREIRA ${ }^{2}$
}

RESUMO - A população de imaturos de Mansonia em águas brancas da várzea da ilha da Marchantaria, na Amazônia Central se constituiu de $74 \%$ de Mansonia (M.) humeralis Dyar \& Knab,1916, 14\% de Mansonia (M.) titillans (Walker, 1848) e 12\% de Mansonia (M.) amazonensis Theobald, 1901, como foi indicado pela frequência, medida por oviposição (posturas). O número de posturas de $M$. humeralis e $M$. titillans foi maior em Pistia stratiotes Linn. enquanto que $M$. amazonensis foi mais abundante em Eichhornia crassipes Solms. O número total de larvas e pupas de Mansonia spp. foi de 6997 e 570 respectivamente, sendo maior em E. crassipes (4372 e 328) que em $P$. stratiotes (2625 e 242). Ao longo do ano, o maior número de larvas e pupas foi encontrado nos meses da vazante (agosto-outubro), tendo um pico no mês de agosto, que não foi correlacionado significativamente com os fatores abióticos.

Palavras-chave: Culicidae; Mansonia; Macrófitas; Várzea; Oviposição.

Oviposition Density and Quantification of Larvae and Puppae of Mansonia Blanchard 1901 (Diptera: Culicidae), in Eichhornia crassipes Solms. and Pistia stratiotes Linn. on Marchantaria Island in the Central Amazon.

ABSTRACT - The population of immature Mansonia in the white-water floodplain of Marchantaria Island, in Central Amazonia was composed by $74 \%$ of Mansonia (M.) humeralis Dyar \& Knab,1916, $14 \%$ of Mansonia (M.) titillans (Walker, 1848) and 12\% of Mansonia (M.) amazonensis Theobald, 1901 , as indicated by frequency of oviposition (egg masses). Number of egg masses of $M$. humeralis and $M$. titillans was higher on Pistia stratiotes whereas that of $M$. amazonensis was higher on Eichhornia crassipes. The total number of larvae and pupae of Mansonia spp. was 6997 and 570 , respectively, and was higher in E. crassipes (4372 and 328) than in P. stratiotes (2625 and 242). Most larvae and pupae were found in the season of lowest water-level (August-October), with a peak in August, not being significantly correlated with abiotic factors.

Key Words: Culicidae; Mansonia; Macrophytes; floodplain; Oviposition

\section{INTRODUÇÃO}

Mansonia Blanchard, 1901 é o gênero de mosquitos hematófagos mais frequente em áreas alagáveis de água branca denominadas várzea (Ferreira, 1994). São encontrados em ambientes florestais anexos à baixadas e alagadiços. Entretanto, isso não impede que possam reproduzir-se nos arredores de cidades, desde que existam condições favoráveis, com locais adequados de criação. O conhecimento do "habitat" desses culicideos constitui um aspecto importante quando se pretende utilizar medidas combinadas para o seu controle. As observações sobre os hábitos das formas imaturas registram que todas as larvas e pupas fixam-se

1 Parte da dissertação, apresentada ao programa de pos-graduação do convênio INPA/UFAM.

2 Instituto Nacional de Pesquisas da Amazônia(INPA) Coordenação de Pesquisas em Entomologia, Caixa Postal 478 - Cep. 69011-970 Manaus, Amazonas, Brasil 
em macrófitas aquáticas (Forattini, 1965). Estas macrófitas ocupam uma posição um tanto ambivalente em ambientes aquáticos, sendo necessárias para um grande número de espécies de animais associadas a elas, possibilitando abrigo e comida. Por outro lado, servem de suporte para espécies vetoras de arbovirus, como é o caso dos imaturos deste gênero.

Neste trabalho foi acompanhado um ciclo anual da densidade de imaturos de três espécies de Mansonia dentro de uma definida vegetação, correlacionando-a com os fatores abióticos como temperatura, precipitação e com o nivel do rio, visando fornecer subsídios para um provável programa de controle desses culicíneos, que agem pela alta densidade , tornando inadequadas certas áreas para colonização.

\section{MATERIAL E MÉTODOS}

\section{Área de Estudo}

O estudo foi realizado na ilha da Marchantaria, uma ilha de várzea no Municipio de Iranduba. Situa-se a $03^{\circ}$ $15^{\prime} \mathrm{S}$ e $59^{\circ} 58^{\prime} \mathrm{W}$ no rio Solimões a 15 $\mathrm{km}$ sudeste de Manaus (BRASIL, 1972). Tem extensão aproximada de 8 x $4 \mathrm{~km}$ de largura, que pode ser aumentada em quase o dobro no período da estação seca (Piedade, 1988).

A ilha da Marchantaria é banhada por águas brancas e possui períodos distintos de inundação (cheia) e seca (vazante). Apresenta um relevo ondulado e a presença dessas ondulações ocasiona a formação de lagos de diferentes dimensões. Alguns lagos têm conexão direta com o rio principal e apresentam seu volume d'água em decorrência do nivel deste (Sternberg, 1956).
A ilha apresenta diferenças sazonais, principalmente com a flutuação do nível das águas e dinâmica das populações de macrófitas aquáticas, que são determinadas pela hidrologia (Piedade, 1988).

\section{Procedimento para as Coletas}

Com base no levantamento dos criadouros potenciais para as espécies de Mansonia, foram selecionadas duas espécies vegetais, identificadas como substrato para fixação dos ovos, larvas e pupas. Para obtenção dos dados foram estabelecidas coletas em locais onde haviam agrupamentos de Eichhornia crassipes em associação biótica, (termo técnico segundo Marino et al., 1980) assim como para Pistia stratiotes.

Os agrupamentos de E. crassipes foram encontrados margeando o lago sem misturar o biótipo grande com o pequeno. $P$. stratiotes ocorre às margens ou nos espaços abertos entre as plantas fixas, ocupando parcelas da água total aberta pela vegetação do lago, podendo também ser encontrada junto ao sedimento nas margens do lago, quando na estação seca.

A escolha dessas espécies foi determinada pelos seguintes fatores: extensas áreas ocupadas por Eichhornia e Pistia na ilha; comprovadas observações da freqüência de Mansonia spp., usando E. crassipes e P. stratiotes como substrato (Dyar \& Knab, 1910, 1916; Costa Lima, 1929; Taylor, 1934; Bidlingmayer, 1968; Gerberg, 1970; Bennett, 1975 e Lounibos \& Escher, 1985); observações e medições já vistas por Jedicke et al. (1989), indicando que ambas as plantas são capazes, sob certas condições, de enriquecer um corpo 
d'água com oxigênio, facilitando desta maneira a sobrevivência de uma enorme fauna associada a elas; e, especialmente, pela oportunidade de poder comparar biótipos de $E$. crassipes e $P$. stratiotes em associação com Mansonia spp.

As amostragens foram feitas no período de 13 meses consecutivos. Os pontos para as amostragens de Mansonia spp. somente foram estabelecidos no dia da coleta. Para isso, o lago foi percorrido em toda sua extensão para a localização de tapetes dessas macrófitas, obedecendo sempre a mesma condição, ou seja, tapete exposto ao sol.

Para a coleta das formas imaturas foram estabelecidos pontos ao longo do lago. Nos agrupamentos de $P$. stratiotes e E. crassipes foram coletadas duas amostras de $1 \mathrm{~m}^{2}$ a cada quinze dias, perfazendo um total de quatro amostras quinzenais por espécie de planta. Cada coleta foi realizada entre 09:00 e 17:00h. Para as amostragens foi utilizado um quadrado de madeira com uma área de $1 \mathrm{~m}^{2}$, que foi lançado sobre os tapetes de macrófitas e as plantas que ficaram dentro foram examinadas e quantificadas. Os espécimes foram coletados com auxilio de uma rede de nylon, que foi passada embaixo da vegetação, trazendo parte das plantas retidas no quadrado. Estas foram então colocadas em bacia esmaltada, retangular, medindo $33 \times 23 \times 5 \mathrm{~cm}$, com água do criadouro. Agitava-se vigorosamente a vegetação para que as larvas e pupas se desprendessem. As raízes foram desfeitas dentro da bandeja e todo o material foi examinado, incluindo os fragmentos que estavam no fundo do recipiente. Repetiu-se essa operação até que se esgotassem as plantas do $\mathrm{m}^{2}$. Os ovos foram retirados junto com a planta à qual estavam aderidos. As larvas e pupas foram então coletadas com uma pequena peneira e colocadas em frascos, fixadas em álcool $70 \%$ e transportadas ao laboratório para posterior triagem e identificação.

\section{Cálculo da Densidade}

A densidade média mensal de posturas das três espécies de Mansonia foi calculada com a seguinte fórmula (Forattini, 1992): DMM = NP / NQ, onde; $\mathrm{DMM}=$ Densidade média mensal; NP = Número total de posturas coletadas de cada espécie em um determinado mês; $\mathrm{NQ}=$ Número de quadrados observados em cada mês.

\section{Análise Estatística dos Dados}

As hipóteses foram avaliadas com testes estatísticos não paramétricos de Kruskal-Wallis e Mann - Whitney ( $\mathrm{p}=$ 0.05 ), e as correlações com o teste não paramétrico de Spearman, Rank Order Correlation $(p=0.05)$ (Zar, 1984). As matrizes dos dados foram feitas no programa "Quattro Pro" 5.0 para Windows 3.11. As análises estatísticas foram realizadas nos pacotes estatísticos Statgraphics 5.0 e em SigmaplotSigmastat 1.0.

\section{RESULTADOS}

O número de posturas das espécies $M$. humeralis e $M$. titillans foi mais abundante em $P$. stratiotes, enquanto que M. amazonensis predominou em $E$. crassipes. Em E. crassipes, a espécie com maior dominância foi $M$. humeralis com $65.9 \%$ da captura, seguida de $M$. amazonensis com $23.3 \%$ e de $M$. 
titillans com $10.8 \%$. Em P. stratiotes, a espécie com dominância significante foi M. humeralis com $79.4 \%$, seguida de $M$. titillans com $16.8 \%$ e de $M$. amazonensis com $3.8 \%$ (Tab. 1). Das duas plantas observadas, o maior número de posturas foi encontrado em $P$. stratiotes; porém, não houve diferença significante no total de posturas das espécies de Mansonia em E. crassipes e $P$. stratiotes, ( $\mathrm{p}=0.877$, Mann-Whitney).

\section{Densidade de Posturas de}

\section{Mansonia spp. em E. crassipes.}

A densidade média de posturas $/ \mathrm{m}^{2}$ de $M$. humeralis variou de 0 posturas/ $\mathrm{m}^{2}$ nos meses de novembro, dezembro de 1991, abril e junho de 1992 a 13,25 posturas no mês de agosto de 1992 . Não houve diferença significante na quantidade de posturas das espécies de Mansonia em E. crassipes, ( $\mathrm{p}=0.08$, Kruskal-Wallis). Para M. amazonensis essa densidade de posturas foi no máximo de 6,5 posturas $/ \mathrm{m}^{2}$ no mês de janeiro de 1992 e a mínima de 0 posturas $/ \mathrm{m}^{2}$ nos meses de novembro de 1991, março, abril, maio e setembro de 1992. M. titillans foi a espécie que teve menor densidade, variando de 0 posturas $/ \mathrm{m}^{2}$ nos meses de novembro e dezembro de 1991, janeiro, fevereiro, abril, maio, agosto e setembro de 1992, a 2,7 em outubro de 1991 (Fig. 1).

\section{Densidade de Posturas de}

\section{Mansonia spp. em P stratiotes.}

A densidade de $M$. humeralis nessa planta foi maior que em $E$. crassipes, variando de 16,25 posturas $/ \mathrm{m}^{2}$, em outubro de 1991 a 0 posturas $/ \mathrm{m}^{2}$, em dezembro de 1991 e janeiro de 1992. Para M. amazonensis a densidade foi menor nessa planta variando de 0 posturas $/ \mathrm{m}^{2} \mathrm{em}$ dezembro de 1991, janeiro fevereiro, março, abril maio, junho e setembro de 1992 a 1 postura $/ \mathrm{m}^{2}$ no mês de novembro de 1991. Em M. titillans, a variação mensal nessa planta foi de 4,2 posturas $/ \mathrm{m}^{2}$, em novembro de 1991 , a 0 posturas $/ \mathrm{m}^{2}$

Tabela 1. Estatística descritiva para o número de posturas das três espécies de Mansonia em E.crassipes e P. stratiotes da ilha da Marchantaria, rio Solimões, durante 13 meses.

\begin{tabular}{|c|c|c|c|c|c|}
\hline Eichhornia crassipes & $x$ & DP & CV & $\%$ & TOT \\
\hline Mansonia (M.) humeralis & 8.5 & 14.1 & 167.2 & 65.9 & 110 \\
\hline Mansonia (M.) amazonensis & 3.0 & 7.0 & 233.3 & 23.3 & 39 \\
\hline Mansonia (M.) titillans & 1.4 & 3.1 & 223.7 & 10.8 & 18 \\
\hline \multicolumn{5}{|l|}{ número de posturas examinadas } & 167 \\
\hline \multicolumn{6}{|l|}{ Pistia stratiotes } \\
\hline Mansonia (M.) humeralis & 13.1 & 18.7 & 143.1 & 79.4 & 170 \\
\hline Mansonia (M.) amazonensis & 0.6 & 1.1 & 182.1 & 3.8 & 8 \\
\hline Mansonia (M.) titillans & 2.8 & 5.7 & 206.5 & 16.8 & 36 \\
\hline \multicolumn{5}{|c|}{ número de posturas examinadas } & 214 \\
\hline
\end{tabular}



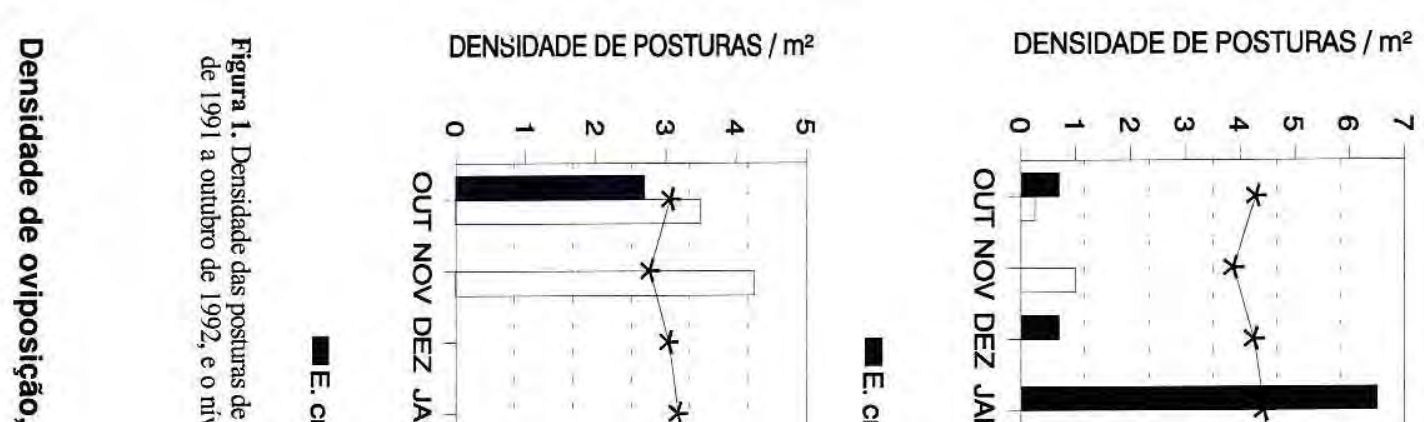

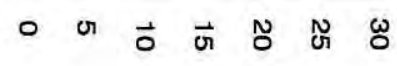

(w) OІ4 OO ר

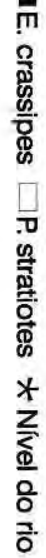

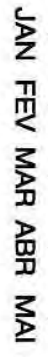
$\frac{c}{z}$

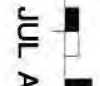
\& 盟 ๖्न

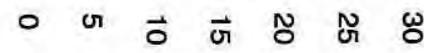

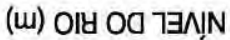

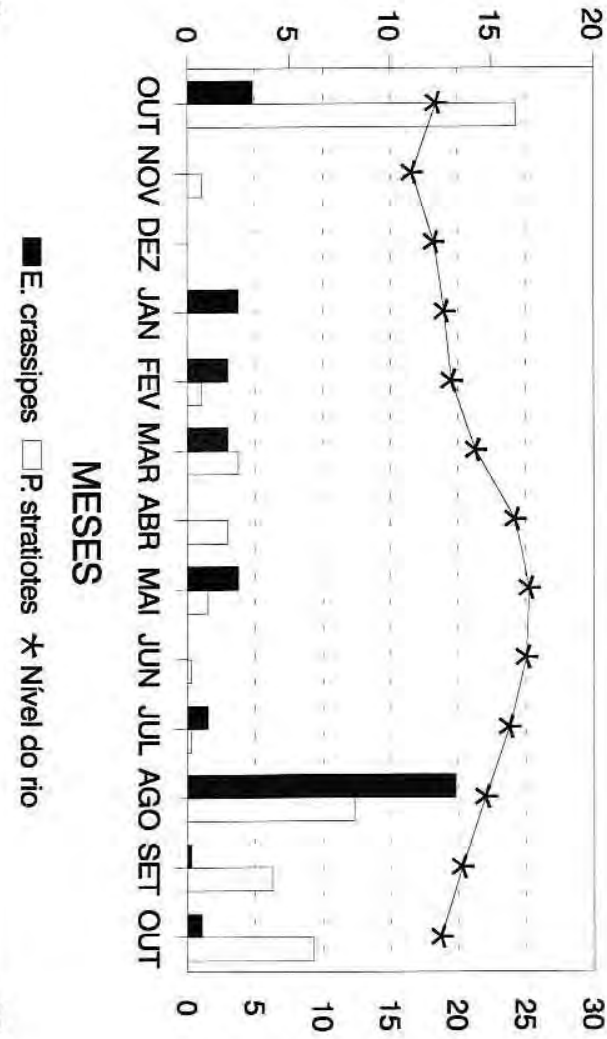

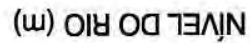


em dezembro de 1991 e janeiro a junho de 1992 (Fig. 1). Entretanto não houve diferença significante na quantidade de posturas das espécies de Mansonia em P. stratiotes $(\mathrm{p}=0.004$, teste de Kruskal-Wallis).

Quando correlacionou-se o número de posturas de Mansonia spp. nas duas espécies de plantas com os fatores abióticos, M. amazonensis em $P$. stratiotes mostrou correlação negativa com a umidade relativa $(\mathrm{r}=-0.67, \mathrm{p}<0.05)$ e precipitação $(\mathrm{r}=-0.63, \mathrm{p}<0.05)$. As posturas de $M$. titillans em $P$. stratiotes apresentaram correlação positiva com a temperatura do ar $(\mathrm{r}=0.61, \mathrm{p}<0.05) \mathrm{e}$ correlação negativa com a umidade relativa $(r=-0.80, p<0.01)$ e com a precipitação $(r=-$ $0.72, \mathrm{p}<0.05)$ (Tab. 3a).

\section{Quantificação de Larvas e Pupas de Mansonia em $\boldsymbol{E}$. crassipes e $\boldsymbol{P}$. stratiotes.}

A quantidade de larvas e pupas de Mansonia spp. nas duas espécies de plantas variou ao longo do ano. $\mathrm{O}$ mês em que mais ocorreram larvas e pupas em E. crassipes e $P$. stratiotes foi agosto de 1992: 1.685 e 1.074 larvas, 123 e 161 pupas respectivamente. Quando comparou-se os números de larvas e pupas coletados em cada espécie de planta, verificou-se que o número em $E$. crassipes foi bem maior que em $P$. stratiotes. Das espécies de plantas observadas, o maior número de larvas foi encontrado em E. crassipes (Tab. 2). Os maiores números de larvas e pupas nas duas espécies de plantas ocorreram durante a época da vazante (Fig. 2).

Não houve diferença significante no número de larvas e pupas de Man- sonia spp. coletadas em E. crassipes $(\mathrm{p}=0.218)$ e $P$. stratiotes. $(\mathrm{p}=0.232)$, teste de Mann-Whitney (Statgraphics 5.0).

Não houve correlação significante entre os fatores bióticos (larvas e pupas das três espécies de Mansonia) em E. crassipes e $P$. stratiotes e os fatores abióticos (temperatura, umidade relativa, precipitação e nivel do rio) (Tab. 3b).

\section{DISCUSSÃO}

O número de posturas de Mansonia spp. coletado em $E$. crassipes e $P$. stratiotes mostrou-se diferenciado. Quando correlacionou-se M.amazonensis. $M$. humeralis e M. titillans nas duas plantas estudadas, não houve significância estatística. Aceitou-se portanto, a hipótese de que não havia diferença no número de posturas colocadas por Mansonia spp. em ambas as plantas. A não significância estatística nas correlações com os fatores abióticos provavelmente é devido ao número insuficiente de exemplares coletados nas duas espécies de plantas (Tab. 2),

Não houve significância ao correlacionar-se o número de posturas de $M$. humeralis em $E$. crassipes com $P$. stratiotes. O mesmo aconteceu para $M$. amazonensis e $M$. titillans quando se correlacionou individualmente essas espécies.

Para $M$. humeralis e $M$. amazonensis não encontramos na literatura informações capazes de esclarecer se existe diferença entre o número de posturas em relação ao substrato. Apesar da não significância estatística, M. titillans parece ser seletiva quanto ao substrato de sustentação para os ovos. Foi descrito por Dunn (1918), 
Tabela 2. Estatistica descrítiva do número de larvas e pupas de Mansonia spp., coletadas em $E$. crassipes e $P$. siratiotes na ilha da Marchantaria, rio Solimões, durante 13 meses.

\begin{tabular}{|c|c|c|}
\hline & $\begin{array}{c}\text { Eichhornia } \\
\text { crassipes }\end{array}$ & $\begin{array}{c}\text { Pistia } \\
\text { stratiotes }\end{array}$ \\
\hline Total de plantas examinadas & 1185 & 1157 \\
\hline $\mathrm{N}^{\circ}$ de plantas $/ \mathrm{m}^{2}$ & 91.15 & 89.00 \\
\hline \multicolumn{3}{|l|}{ Larvas } \\
\hline $\mathrm{N}^{\circ}$ de individuos $/ \mathrm{m}^{2}$ & 336.3 & 201.9 \\
\hline$N^{\circ}$ de individuos / planta & 3.7 & 2.3 \\
\hline DP & 464.4 & 325.2 \\
\hline $\mathrm{CV}$ & 138.1 & 161.0 \\
\hline TOT & 4372 & 2625 \\
\hline$\%$ & 62.5 & 37.5 \\
\hline \multicolumn{3}{|l|}{ Pupas } \\
\hline $\mathrm{N}^{\circ}$ de individuos $/ \mathrm{m}^{2}$ & 25.2 & 18.6 \\
\hline $\mathrm{N}^{\circ}$ de individuos / planta & 0.27 & 0.20 \\
\hline DP & 35.7 & 43.7 \\
\hline CV & 141.3 & 234.8 \\
\hline TOT & 328 & 242 \\
\hline$\%$ & 57.5 & 42.5 \\
\hline
\end{tabular}

que imaturos de $M$. titillans têm sido coletados quase que exclusivamente em $P$. stratiotes. Isso difere um pouco dos resultados do presente trabalho onde se observou posturas desse culicídeo nas duas espécies de plantas estudadas.

Informações da literatura e observações no campo mostraram que as fêmeas de Mansonia spp. depositam massas de ovos sob folhas de macrófitas aquáticas. Assim, apesar do número de posturas de $M$. titillans coletadas em $E$. crassipes e $P$. stratiotes, não terem apresentado significância nas correlações, as posturas mais abundantes de $M$. titillans foram em $P$. stratiotes. É provável que esta preferência seja explicada pelo fato da folha de $P$. stratiotes possuir uma superficie pilosa oferecendo melhor proteção contra a dessecação. Ao contrário, a folha de $E$. crassipes possui uma superficie lisa.

As correlações de Spearman apresentaram significância entre as posturas de $M$. titillans em $P$. stratiotes com a temperatura (correlação positiva), com umidade relativa e com a precipitação (correlação negativa) e M. amazonensis em $P$. stratiotes com umidade relativa e com a precipitação (correlação negativa) (Tab. 3a). $M$. humeralis ao contrário, não mostrou correlação significante com esses fatores, sendo aparentemente menos sensivel, o que poderia explicar a maior abundância dessa espécie na ilha.

$\mathrm{O}$ ano equivalente ao período de coleta apresenta-se atípico em relação ao 


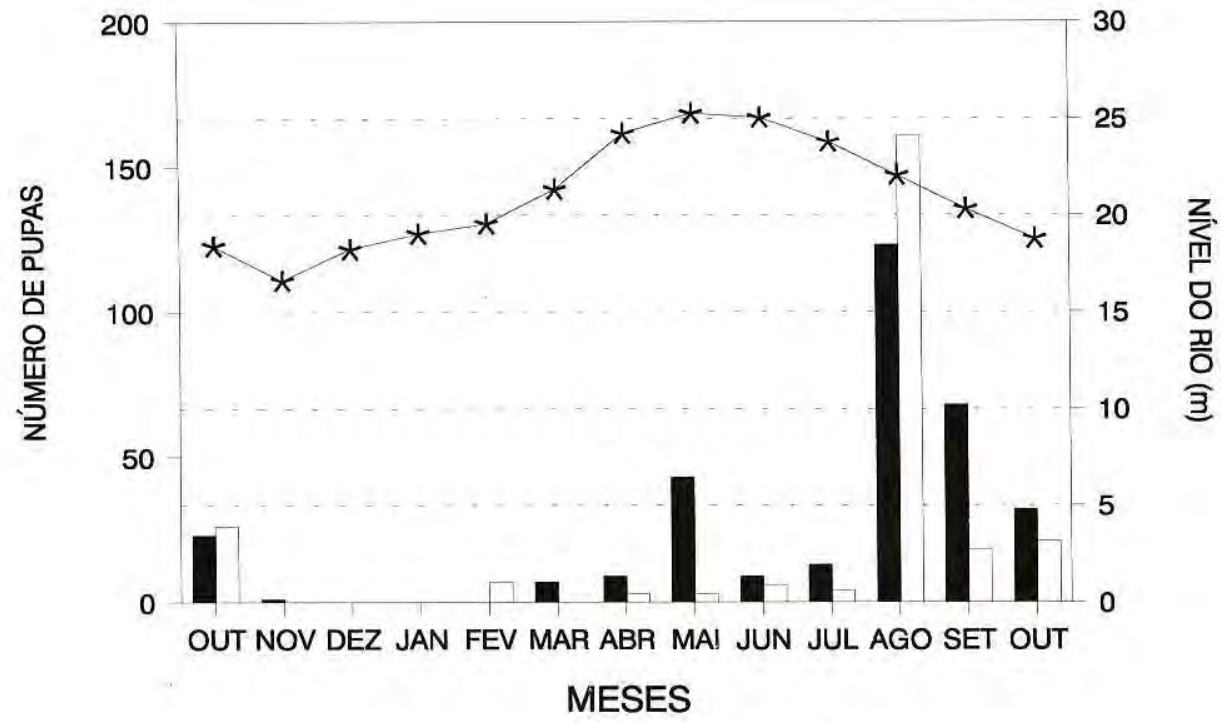

E. crassipes $\square$ P. stratiotes $\star$ Nível do rio

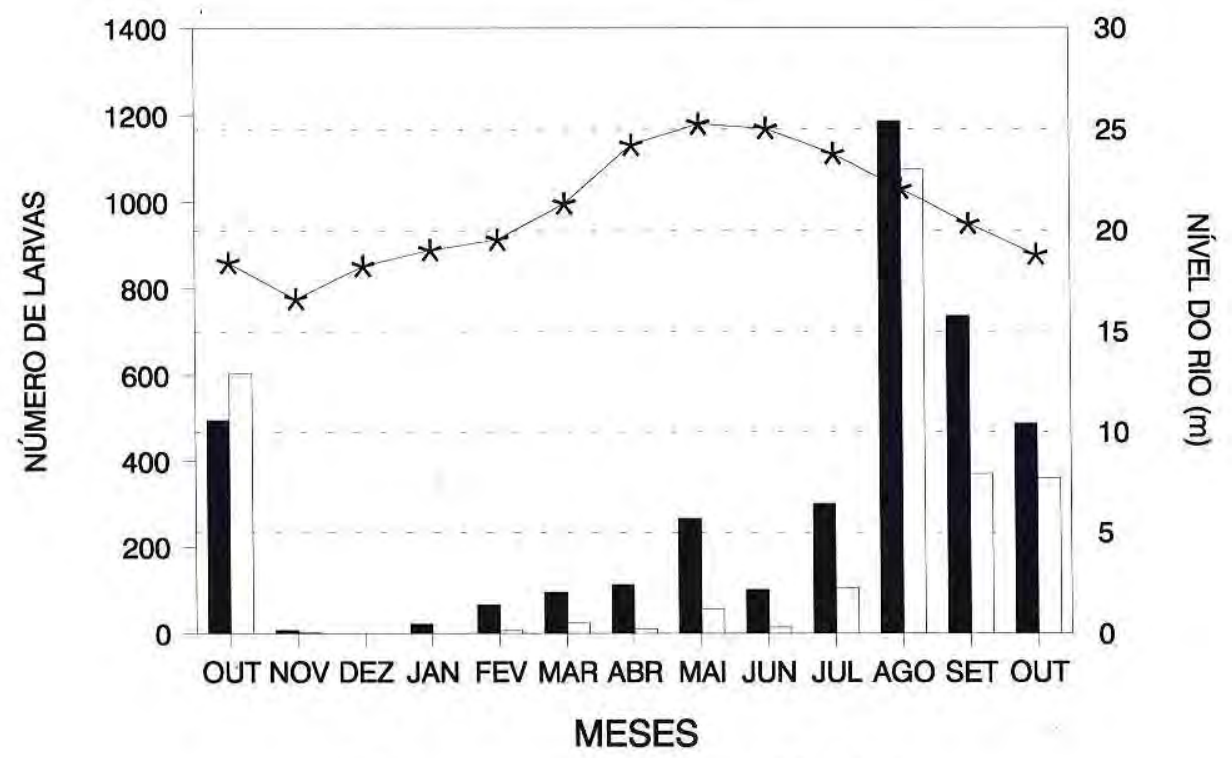

E. crassipes $\square$ P. stratiotes $\star$ Nível do rio

Figura 2. Número de larvas e pupas de Mansonia spp. em e. crassipes e $P$. stratiotes e o nível do rio, na ilha da Marchantaria, rio Solimões. 
Tabela 3. Coeficiente da correlação de Spearman entre a. o número de posturas das três espécies de Mansonia em E. crassipes e $P$. stratiotes com os fatores abióticos e b. o número de larvas e pupas de Mansonia spp. em E. crassipes e $P$. stratiotes com os fatores abióticos.

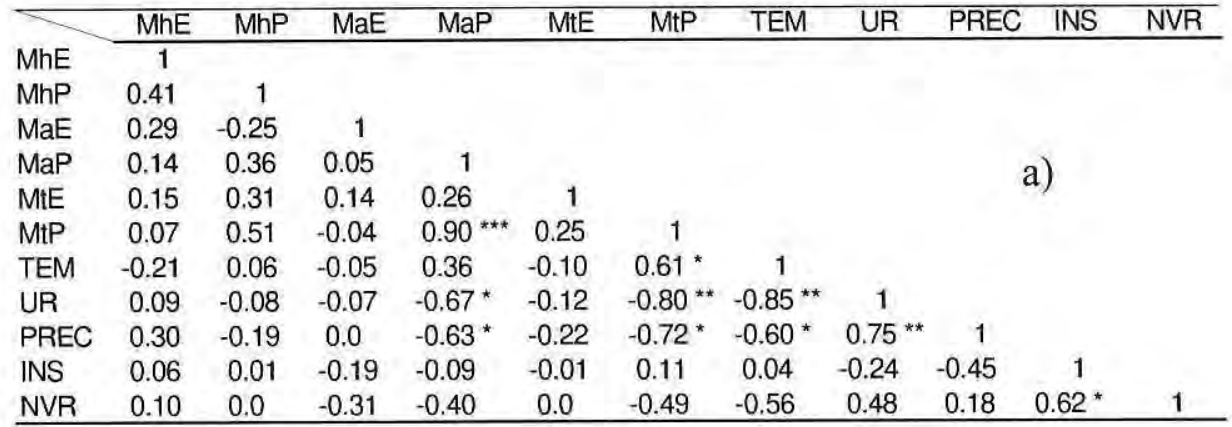

${ }^{*}=p<0.05$

$\mathrm{MhE}=$ M.humeralis/E.crassipes

MhP $=$ M.humeralis/P.stratiotes

TEM = Temperatura

$\mathrm{UR}=$ Umidade relativa

$$
{ }^{\star *}=p<0.01
$$

$\mathrm{MaE}=$ M.amazonensis/E.crassipes $\mathrm{MaP}=$ M.amazonensis $/$ P.stratiotes PREC $=$ Precipitação

INS = Insolação

$$
\begin{aligned}
* * * & =p<0.001 \\
\text { MtE } & =\text { M.titillans/E.crassipes } \\
\text { MtP } & =\text { M.titillans/P.stratiotes } \\
\text { NVR } & =\text { Nível do rio }
\end{aligned}
$$

LCRAS 1.00

LPSTRAT 0.86001 .00

\begin{tabular}{lrrrrrrrrr} 
PCRAS & 0.8161 & 0.7684 & 1.00 & & & & & b) \\
PSTRAT & 0.7885 & 0.7936 & 0.7101 & 1.00 & & & & \\
TEMP & 0.0790 & 0.0397 & 0.1216 & 0.071 & 1.00 & & & \\
UMR & -0.1438 & -0.1842 & -0.1477 & -0.2257 & -0.7383 & 1.00 & & \\
PREC & -0.1795 & -0.2194 & -0.2369 & -0.4288 & -0.4738 & 0.5883 & 1.00 & \\
INS & 0.2308 & 0.1936 & 0.3422 & 0.2905 & 0.0526 & -0.1700 & -0.3300 & 1.00 \\
NVR & 0.2564 & 0.1677 & 0.2896 & 0.1245 & -0.3949 & 0.3269 & 0.1500 & 0.4600 & 1.00 \\
\hline
\end{tabular}

NVR

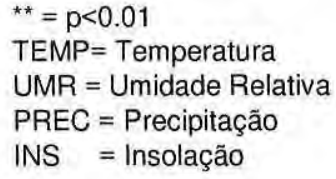

NVR $=$ Nivel do rio nível do rio (Fig. 3). Contudo, é importante mencionar as interligações que existern entre os decursos da temperatura, umidade relativa, precipitação e do nível do rio (Fig. 4). Quando a temperatura sobe, a umidade relativa e precipitação baixam e vice versa. Isso explica a correlação negativa significante entre umidade relativa e temperatura, coincidindo com a correlação positiva significante entre umidade relativa e precipitação. Na Amazônia, a distribuição da precipitação durante o ano não é homogênea. A precipitação não coincide com o nível do rio, mas mostra um adiantamento da fase na ondulação anual (Junk, 1983). Isto se verifica também para o período estudado (Fig. 4).

As flutuações do nivel da água têm 


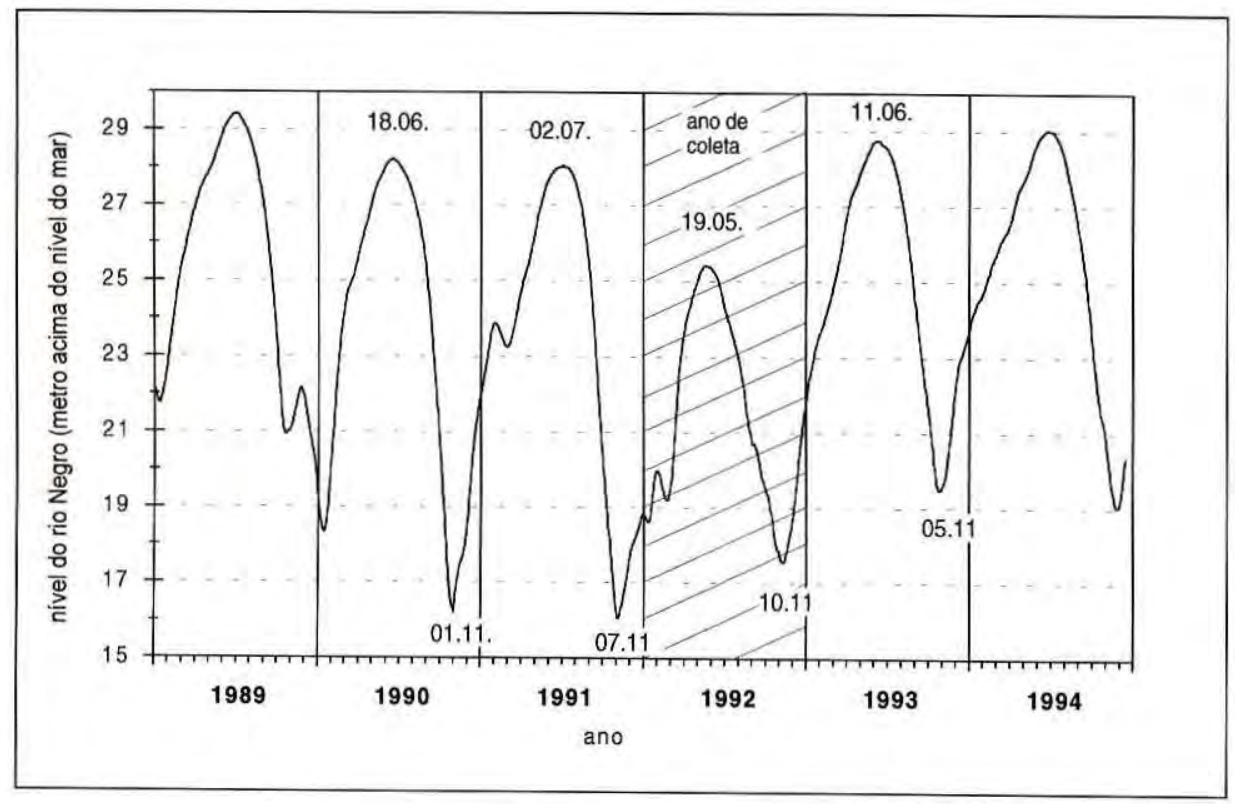

Figura 3. Variação anual do nivel do rio Negro, no período de 1989 a 1994, com destaque para 1992. (Dados fornecidos pela Capitania do Porto de Manaus).

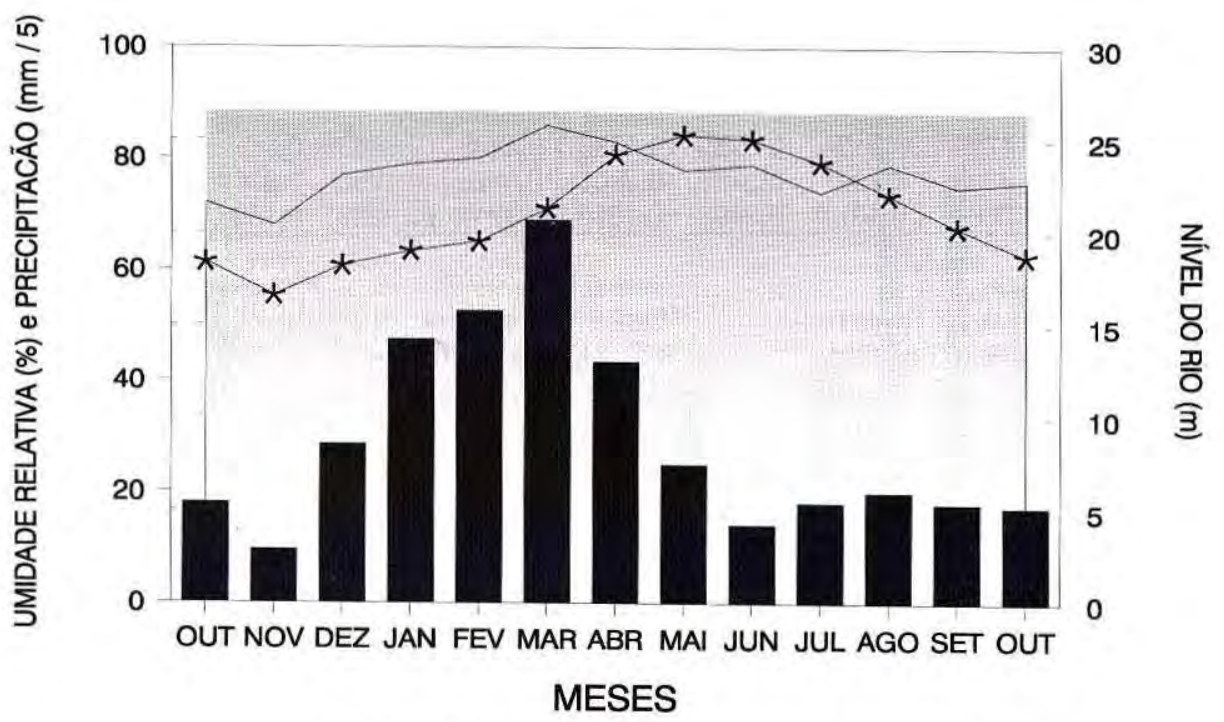

$\square$ Umidade Relativa Erecipitaçāo * Nível do rio

Figura 4. Variação do nivel do rio Negro, precipitação e umidade relativa no periodo de outubro 1991 a outubro 1992. 
efeito importante para a fauna da várzea. Apesar de não ter tido correlação significante com o nivel do rio, os dados da Figura 1 sugerem uma tendência a um aumento de posturas na vazante.

Não houve diferença significante no número de larvas e pupas de Mansonia coletadas em E. crassipes e $P$. stratiotes, assim como na abundância média de larvas e pupas em $E$. crassipes e P. stratiotes. Porém, quando se comparou o número de larvas e pupas coletadas em cada espécie de planta, verificou-se que o número em $E$. crassipes foi bem maior do que em $P$. stratiotes.

Levando-se em consideração que $P$. stratiotes seja o melhor criadouro para as posturas, surpreende-se que foi coletado o maior número de larvas e pupas em E. crassipes. No entanto, Jedicke et al. (1989) revelam que as raizes de E. crassipes liberam mais oxigênio que $P$. stratiotes. Isto pode ser uma explicação para essa preferência. Quando correlacionou-se a quantidade de larvas e pupas com os fatores meteorológicos e o nível do rio, não se observou resultados significantes (Tab. 3b). Aparentemente estes fatores não tiveram influência sobre as larvas e pupas nas plantas. Apesar da não-correlação entre a quantidade de larvas e pupas com o nivel do rio, os dados da Figura 2 sugerem uma certa tendência no aumento de larvas e pupas na vazante.

\section{AGRADECIMENTOS}

Ao Dr. José Alberto S. Nunes de Mello pelos recursos concedidos através do Instituto Max-Planck de Limnologia, Plön-Alemanha Projeto
INPA/MPI e ao José Camilo H. Guerreiro pela grande ajuda na análise estatística dos dados.

\section{Bibliografia citada}

Bennett, F.D. 1975. Insects and plant pathogens for the control of Salvinia and Pistia. In: Brezoniz, P. L.; Fox, J. L. (eds.) The Proceedings of a Symposium on water quality management through biological control, jan. (23-30). Gaineswille, Florida: 28-35.

Bidlingmayer, W.L. 1968. Larval development of Mansonia mosquitoes in central Florida. Mosq. News, 26(1): 51-57.

BRASIL 1972. Ministério das Minas e Energia DNPM. Carta imagem de Radar, folhas Sa. 20 Z-D e Sa. 21-Y-C (1:250.000). Brasilia.

Costa Lima, A. 1929 Sobre algumas espécies de Mansonia encontradas no Brasil. Supl.Mem.Inst.Oswaldo Cruz,12: 297- 300.

Dunn, L.H. 1918. The lake mosquito Mansonia titillans Walk, and it's host plant, Pistia stratiotes, Linn., in the canal zone, Panamá (Diptera: Culicidae). Entomol.News, 29: 288-295.

Dyar, H.G.; Knab, F.1910. The genus Mansonia. Entomol. News and Proc. of the Entomological, section of the Academy of Natural Sciences of Philadelphia, 21: 259-264.

.... 1916. Eggs and oviposition in certain species of Mansonia (Diptera: Culicidae). Insecutor Inscitiae Menstruus. A Monthly Journal of Entomology, 4(1-3): 61-68.

Ferreira, R.L.M. 1994. Aspectos Biológicos de Mansonia Blanchard, 1901 (Diptera: Culicidae) da ilha da Marchantaria, rio Solimões, Amazonas. Dissertação de Mestrado. INPA/ FUA. Manaus-Amazonas.108p.

Forattini, O.P. 1965. Entomologia Médica. Vol. 3. Culicini: Haemagogus, Mansonia, Culiseta. Sabethini, Toxorhynchitini, Arboviroses, Filariose bancroftiana, Genética. Universidade de São Paulo, 416 p.

1992. Ecologia, Epidemiologia e Sociedade. São Paulo: Artes Médicas: Editora da Univerdade de São Paulo. III - 529 p.

Gerberg, E. J. 1970. Manual for mosquito 
rearing and experimental tecniques. Amer.Mosq.Control.Ass.Inc., 5: 47-49.

Jedicke, A.; Furch, B.; Saint-Paul, U.; Schlueter, U.B. 1989 Increase in the oxygen concentration in Amazon waters resulting from the root exudation of two notorious water plants, Eichhornia crassipes (Pontederiaceae) and Pistia stratiotes (Araceae). Amazoniana, 11(1): 53-69

Junk, W.J. 1983. As Águas da Região Amazônica; In: Salati E.; Schubart, H.O.R., Junk, W.J., Oliveira, A.E. de (eds). Amazonia. CNPq, Editora Brasiliense: p. 45-100.

Lounibos, L.P.; Escher, R.L. 1985. Mosquitoes associated with water lettuce (Pistia stratiotes) in Southeastern Florida. The Florida Entomologist, 68(1):169-178.

Marino, M. C.; Furtado J.S.; Vuono Y.S. de 1980. Glossário de Termos Usuais em Ecologia. 159p. 1" edição. Publicação ACIESP $n^{\circ}$ 24/Academia de Ciências do Estado de S. Paulo.
Piedade, M. T. F. 1988. Biomassa, produtividade e atividade fotossintética de Echinochloa polystachya (H. B. K.) Hitchcok (Graminae = Poaceae), capim-semiaquático da várzea Amazónica. Tese de Doutorado, pelo Convênio do Instituto Nacional de Pesquisas da Amazônia com a Universidade Federal do Amazônas INPA/FUA. ManausAmazonas. $154 \mathrm{p}$.

Stemberg, H.O. 1956. A água e o homem na várzea do Careiro. Tese de Concurso a categoria de Geografia do Brasil da Faculdade Nacional de Filosofia da Universidade do Brasil, Rio de Janeiro, $229 \mathrm{p}$.

Taylor, W.P. 1934. Notes on the water lettuce, Pistia stratiotes Linn., as a Nursery of insect life. Ecology, 15(3): 328-331.

Zar, J. 1984. Biostatistical Analysis Prentice. Hall. Second Edition. 717 p. 